\title{
EDITORIAL
}

\section{El “ideal realista” europeo. Lo que está en juego en las elecciones al Parlamento europeo}

\section{Consejo de Redacción}

Resumen: Unas elecciones como las del 25 de mayo de 2014, convocadas para elegir un nuevo Parlamento europeo para los próximos años (2014-2019), son un momento esencial en toda organización democrática. El Parlamento europeo ejerce un control "democrático" sobre la Comisión y, por primera vez, elegirá formalmente al presidente de ésta. En estas elecciones se manifiesta la existencia de una ciudadanía europea, con sus logros y también con todas las dificultades y retrasos para su construcción. Por otro lado una parte importante de esos ciudadanos o son escépticos o militan contra el europeísmo, pero, como es lógico, votan y lograrán una parte quizá bastante importante, de la representación parlamentaria. Junto a la ciudadanía está la existencia de unos políticos europeos y la burocracia que les presta servicio, además de la dimensión económica en juego en relación con la moneda única. El editorial concluye con una mirada mundial desde la perspectiva europea. Hasta hace poco Europa era un modelo de referencia mundial, y probablemente para muchos sigue siéndolo, lo que exige que una reflexión política sobre la Europa actual deba hacerse siempre en una perspectiva global.

Palabras clave: Parlamento europeo, ciudadanía europea, control democrático, euro, valores europeos.

Fecha de aprobación: 13 de febrero de 2014. 
The European realistic ideal. What is at stake in the elections to the Parliament of the European Union

Abstract: Some elections as the of the 25 of may of 2014, convened to elect a new European Parliament for nextyears (2014-2019), are essential in any democratic organization time. The European Parliament exercises a 'democratic' control over the Commission and, for the first time formally elect the President of this Commission. These elections manifests the existence of European citizenship, with their achievements and also with all the difficulties and delays for its construction. On the other hand an important part of those citizens, or they are skeptical or they militate against the Europeanism, but, surprisingly, they vote and achieve some perhaps quite important, parliamentary representation. Next to citizenship is the existence of some European politicians and the bureaucracy that serves them, as well as the economic dimension at stake in relation to the single currency. The editorial concludes with a world view from a European perspective. Until recently Europe was a global reference model, and probably for many it still is, which requires that a political reflection on today's Europe should always be done in a global perspective.

Keywords: European Parliament, European citizenship, democratic control, euro, European values.

\section{L'idéal européen réaliste. Ce qui est en jeu lors des élections au Parle- ment de I'Union européenne}

Résumé: Des élections comme celles du 25 mai 2014, convoquées pour élire un nouveau Parlementeuropéen pour les années prochaines (2014-2019), sont un élément essentiel dans n'importe quel moment d'una organisation démocratique. Le Parlement européen exerce un contrôle "démocratique» au dessus-de la Commission et, pour la première fois formellement, il doit élire son Président. Dans cettes elections on manifeste l'existence d'une citoyenneté européenne, avec leurs réalisations ainsi qu' avec toutes les difficultés et les retards pour sa construction. D'autre côté, une partie importante de ces citoyens, ou ils sont sceptiques ou ils militent contre l'européanisme, mais ils votent et ils attiendron peut-être assez une importante représentation parlementaire. À côté de la citoyenneté est ily sont certains responsables politiques européens et la bureaucratie qui est au son service, ainsi que la dimension économique à jalonner par rapport à la monnaie unique. L'éditorial conclut avec une vision du monde dans une perspective européenne. Jusqu'à ce que récemment l'Europe a été un modèle de référence mondiale, et probablement pour beaucoup il en l'est toujours, ce que exige qu'une réflexion politique sur l'Europe d'aujourd'hui doit toujours se faire dans une perspective globale.

Mots clés: Parlement européen, citoyenneté européenne, contrôle démocratique, euro, valeurs européennes. 


\section{Introducción}

La convocatoria de elecciones constituye por definición el momento "mágico" decisivo en toda comunidad organizada democráticamente. Sin embargo parece que las elecciones europeas plantean a menudo ciertas dudas e interrogantes para el ciudadano: el Parlamento europeo nos resulta una institución lejana, dotada de competencias algo difusas o evanescentes, que además sufre, como el resto de las instituciones de la Unión Europea, el permanente y reiterado desgaste de unas críticas referidas al famoso "déficit democrático". También suele aludirse a la inexistencia de un auténtico "poder legislativo", dado que la normativa europea sigue un proceso de elaboración que no es equivalente a las tradicionales leyes de los parlamentos nacionales; así como al hecho de que, en la práctica, el auténtico brazo ejecutor de la política europea parece ser más bien la Comisión'.

Pero a pesar de todas estas críticas, debemos recordar que el Parlamento es el órgano encargado de controlar políticamente a la Comisión, y que, después del famoso caso Santer en 1999, el desarrollo de esa labor de control se ha consolidado progresivamente, hasta el punto de permitir una dinámica de tipo parlamentario que refuerza en la práctica el papel de la cámara europea tanto en términos de confianza política como de control efectivo sobre la actuación de la Comisión.

Precisamente aquí radican las principales novedades que, tras el Tratado de Lisboa de 2007, se ponen en marcha en las elecciones del próximo mes de mayo: por una parte un protagonismo directo de la cámara en el nombramiento del presidente de la Comisión a través de una votación de investidura; por otra, la obligación de los partidos de presentar a sus cabezas de lista como potenciales candidatos a tal puesto. Además, los partidos nacionales tendrán que informar durante la campaña a qué partido europeo están adscritos así como a qué candidato van a apoyar para la presidencia de la Comisión. Este último punto tiene, desde un punto de vista político, una considerable importancia ya que permite teóricamente a los electores "visualizar" a quien será, o quisieran que fuese, futuro presidente de la Comisión.

Pero una convocatoria electoral se supone que en primer lugar es, o debe ser, un momento adecuado para realizar un balance, para reflexionar acerca de la actua-

' El proceso de elaboración de este editorial, como en otras ocasiones, tuvo su punto de partida en un "brain storming" del Consejo de redacción de RFS sobre el tema de las elecciones europeas de mayo de 2014. Un primer borrador fue elaborado por un miembro de nuestro consejo. Dicho texto fue revisado por otros miembros del consejo y aprobado por el mismo, dando lugar a la versión que presentamos. 
ción general de la Unión Europea durante los últimos cinco años ${ }^{2}$. Y en este sentido no cabe sino constatar que durante la reciente gran crisis económica la capacidad de respuesta de las instituciones europeas no se ha caracterizado precisamente por su rapidez, eficacia y contundencia: las incertidumbres que han rodeado las políticas de rescate a ciertos países, las ambigüedades en que se ha movido a veces el Banco Central, Europeo (en parte debidas a las limitaciones a las que se encuentra sometido, debido a las normas que regulan su actuación) la sensación ocasional de desorientación o de impotencia, serían síntomas no sólo de un cierto déficit de capacidad reflexiva en el seno de las instituciones europeas, sino también de la insuficiente "madurez" institucional de las mismas, en el sentido de que, en la práctica, han sido a veces las decisiones de los estados (y en particular de uno de ellos, Alemania) las que han marcado la pauta en las principales decisiones, desbordando así a la propia Unión.

¿Es el actual un momento para la refundación de la UE? Suele decirse en todo caso, en un sentido constructivo, que los momentos de crisis son, o pueden ser al mismo tiempo, momentos creativos, donde las instituciones se enfrentan, como un ave fénix, a los retos de una suerte de "refundación" capaz de reforzar su capacidad general de liderazgo y de respuesta a los problemas sociales.

Es cierto que la necesidad se convierte en virtud y no cabe ignorar la dimensión de las respuestas ante la crisis. En el año 2010 se crearon una serie de autoridades

\footnotetext{
${ }^{2}$ No abordamos el tema europeo por primera vez, ni mucho menos. En los últimos años nuestra revista se ha posicionado de forma recurrente en sus reflexiones editoriales acerca del dinámico proceso de la construcción europea y de algunos aspectos relacionados con el mismo. Tomando una referencia temporal significativa, recordamos que -a partir del año 2000- nos hemos pronunciado casi una docena de veces: "La política de la Unión Monetaria Europea y sus consecuencias para España": RFS 67 (2012) 573-606, n 268; "Un sistema de economía social de mercado para una Europa solidaria, responsable y productiva": RFS 67 (2012) 5-29, n 265: "El futuro de la Política Agraria Común Europea: nuevas perspectivas": RFS 66 (2011) 155-179, n 262; "La crisis del Euro": RFS 65 (2010) n² 260; "'La ilusión de la identidad': el actual debate europeo": RFS 64 (2010) 13-31, n 257; "La Constitución Europea renace de sus cenizas: el Tratado de Lisboa":RFS 62 (2008) 11-29, n 249: "Claves para comprender la crisis financiera internacional": RFS 61 (2007) 515-535, n 248; "La financiación de la Unión Europea: los límites de una ambición": RFS 60 (2006) 139-164, n²42; "Una reflexión sobre la construcción del espacio de educación superior": RFS 59 (2005) 387-405, n 239; "Más allá de la Constitución Europea: Europa entre raíces cristianas y laicidad": RFS 58 (2004) 727-749, n 236; "La Política Agraria Común en un mundo globalizado": RFS 55 (2001) 343-367, $n^{\circ} 223$. Si volvemos ahora sobre el tema es por dos razones: la primera, porque la idea de Europa se encuentra actualmente en una grave crisis, quizá la más grave de su historia; la segunda, porque la proximidad de las elecciones al Parlamento Europeo del 25 de mayo próximo ofrece una ocasión propicia para plantear la cuestión. En esta complicada coyuntura, deseamos pronunciarnos acerca de lo que podríamos denominar el "ideal realista" de Europa.
} 
independientes con tareas de control y de vigilancia ante los riesgos de crisis sistémica (la "European Systemic Risk Board", junto a las nuevas autoridades independientes: Autoridad Europea de Valores y Mercados -"European Securities and Markets Authority"-, Autoridad Europea de Seguros y Pensiones de Jubilación -"European Insurance and Occupational Pensions Authority"-, y Autoridad Bancaria Europea -"European Banking Authority"-). La serie se continuó con el llamado "Six Packs" de 2011 (cinco Reglamentos y una Directiva) y dos Reglamentos de mayo 2013 ("Two Packs"): todo ello en el marco del ordenamiento de la Unión.

Otras iniciativas se han formulado fuera del propio derecho de la Unión, en forma de tratados internacionales, como el Tratado Constitutivo del Mecanismo Europeo de Estabilidad (febrero 2012) o el Tratado de Estabilidad, Coordinación y Gobernanza en la Unión Económica y Monetaria (marzo 2012).

Sin embargo el balance de resultados de todas estas iniciativas parece todavía algo prematuro $y$, por otra parte, no parece que la capacidad de liderazgo manifestada hasta ahora por Barroso o van Rompuy haya conseguido consolidar el papel de la Comisión o del Consejo; de tal forma que un balance realista de sus actuaciones nos ofrecería un panorama final bastante incierto. La idea de que, tras las próximas elecciones europeas, y en un espíritu de renovación frente a la crisis, pueda llegar a consolidarse una nueva y original sinergia creativa de la Unión hasta el punto de suscitar una dinámica institucional reforzada y apoyada por la ciudadanía europea, constituye evidentemente un deseo optimista que merecería ser apoyado. Aunque sin perder de vista también las crecientes amenazas del desencanto, acompañado de la aparición de fuerzas populistas o xenófobas y el desarrollo general de un cierto euroescepticismo que puede acabar debilitando aún más el proyecto europeo.

Estamos ante un momento de pérdida de cohesión y de aumento de la dialéctica norte/sur. Mucho más grave resulta sin embargo el efecto colateral o indirecto que han generado las consecuencias de la crisis en términos de cohesión a escala europea: se ha producido, desde esta perspectiva, un desencadenamiento de la tensión norte/sur frente a la cual, teóricamente, se habían ido desplegando durante las décadas anteriores todo un sinfín de políticas de cohesión que constituían seguramente el entramado más precioso de la política europea. Es decir, todo un esfuerzo consciente cohesión social o de nation building (por utilizar una expresión clásica) que, sin embargo, ha acabado manifestando una dramática precariedad que al final está impactando gravemente contra los sectores sociales más débiles de la Europa mediterránea. Suscita una gran tristeza constatar que aquello que considerábamos como el más brillante logro europeo, la generación de políticas 
de integración y cohesión social en una escala transnacional, haya demostrado tal grado de fragilidad que nos retrotrae a épocas históricas muy pretéritas, cuando las tensiones entre el norte europeo industrializado y las débiles y atrasadas sociedades agrarias del sur constituían el habitual marco geopolítico de referencia para analizar la realidad de nuestro viejo continente.

\section{La dimensión política. El sentido de unas elecciones europeas}

Pero comencemos por el principio: se supone que una convocatoria electoral es en primer lugar un acontecimiento de tipo político; probablemente el más "político" de los momentos a los que se enfrenta un ciudadano normal en cualquier sociedad democrática. En su expresión más profunda se trata de una manifestación activa del "estatus" de ciudadanía, en este caso de la ciudadanía europea, lo que implica dar por supuesta una condición de pertenencia colectiva; es decir, la sensación subjetiva de ser miembros de una comunidad que comparte ciertos valores comunes y participa en un proyecto igualmente común.

La ciudadanía europea es expresión de la pertenencia en una realidad compleja multinivel. Naturalmente cuando, como ciudadanos, nos enfrentamos ante una realidad institucional compleja, caracterizada por su proyección en una escala multinivel (es decir, cuando pertenecemos a esferas institucionales públicas de distinta proyección territorial, como mínimo la local, la regional o autonómica, la estatal y la europea), esta sensación de pertenencia puede quedar en parte diluida o "deconstruída". Y la pregunta inmediata es si realmente en el año 2014 nos sentimos lo suficientemente "europeos" como para comprometernos colectivamente con el sentido de una decisión que debemos expresar a través del voto. En este punto parece correcta la afirmación de que a veces el llamado "déficit democrático" de la UE es simplemente un problema de lejanía perceptiva: a las instituciones europeas no podemos pedirle el mismo grado de inmediatez y de visualización que le pedimos normalmente a un ayuntamiento; y por otra parte, las identidades colectivas no siempre tienen por qué implicar un alto grado de identificación de tipo "comunitarista", sobre todo en un contexto mundial donde los flujos migratorios parecen desbordar todos los cauces preestablecidos. Bastaría entonces con recordar que el derecho de sufragio es simplemente un derecho legalmente adquirido, propio de todo sistema democrático, que nos otorga una capacidad para influir en la agenda y en las decisiones de las instituciones representativas correspondientes. 
La primera expresión legítima que puede adquirir el sufragio es la de ser un instrumento de protesta, es decir, en nuestro caso, un mecanismo a través del cual muchos ciudadanos pueden expresar su malestar, a modo de respuesta frente a una crisis que ni nadie supo prever a tiempo ni acaso está siendo gestionada adecuadamente desde las esferas europeas. El voto, no cabe duda, puede ser una expresión de malestar social como lo ha sido ya en algunas elecciones nacionales y de ahí que no quepa sorprenderse excesivamente ante la aparición de nuevas fuerzas o candidaturas de carácter crítico, populista o radical.

Otra cosa distinta es que el antieuropeísmo constituya en rigor una respuesta adecuada ante la situación actual: y no sólo por la irreversibilidad de ciertos procesos históricos sino porque fuera de la Unión Europea el grado de riesgo o de incertidumbre con el que los estados pueden enfrentarse a situaciones de crisis se incrementaría dramáticamente.

Sin embargo una posición crítica expresada en su caso a través del voto no tiene por qué traducirse en una manifestación de rechazo radical a la existencia misma - a la pertenencia a la UE, sino que puede también constituir la expresión de una voluntad de reorientación o de reformas en las propias instituciones europeas.

Puede comenzarse recordando que el principal obstáculo al desarrollo efectivo del proceso europeo en los últimos años han sido, paradójicamente, los gobiernos de los propios estados miembros. Víctimas seguramente de un miedo al vacío que puede suponer una pérdida de poder, los gobernantes estatales no han permitido, ante el complejo contexto de la crisis, un reforzamiento efectivo de la capacidad de decisión de las instituciones europeas: hemos comprobado cómo las decisiones de los gobiernos se interponían o interferían con las decisiones de las propias instituciones de la Unión. Y lógicamente el resultado ha sido que los gobiernos más poderosos, y más en concreto el gobierno alemán, han sido los que han acabado dirigiendo en la práctica la difícil política anticrisis. Un protagonismo que no siempre ha estado guiado por los intereses generales de la propia Unión. Se trata de una apuesta que ha respondido por otra parte a claves constitucionales internas, pues ha sido el tribunal constitucional alemán el encargado en última instancia de dar el visto bueno a las distintas decisiones, manteniendo siempre el criterio de que los valores constitucionales del propio estado son, en cierta manera, "superiores" a los de la propia Unión.

De ahí que la pregunta sobre quién manda realmente en Europa pueda adquirir un cierto sentido, ya que el debilitamiento relativo de las instituciones europeas provoca un desigual poder de influencia por parte de los distintos estados: y la 
impronta dominante germana, tan influida por sus propias pautas culturales y por la ideología de sus actuales gobernantes, parece que conduce a declarar a los países del sur como "culpables" de la crisis, que ahora estamos purgando con todas sus consecuencias. Otras posiciones estratégicas, como las que se defienden desde algunos medios académicos norteamericanos, proponiendo medidas menos restrictivas frente a la crisis, no acaban de encontrar entonces un eco suficiente en las esferas europeas.

Sin embargo resulta paradójico que, al mismo tiempo que el contexto de la actual crisis ha estimulado el euroescepticismo y el reverdecimiento de un sentimiento nacionalista que contribuye a reforzar la dimensión intergubernamental de la Unión Europea y su concepción como una mera unión internacional de estados, paralelamente los instrumentos jurídicos y económicos de respuesta a la misma están profundizando en algunos aspectos el proceso de integración, reduciendo la autonomía parlamentaria de los estados en ámbitos tan decisivos como la aprobación de sus presupuestos o de sus respectivas políticas económicas, llevando así hasta el límite su presión sobre las estructuras constitucionales de los respectivos estados miembro.

Pero se critica también que la burocracia de Bruselas, sin duda integrada por unos cuadros dotados de excelente preparación técnica, suele estar muy influida por la presencia de "lobbies" o grupos de presión que representan intereses poderosos con gran capacidad de influencia en las decisiones de la Unión, imponiendo así un alejamiento de los intereses ciudadanos. Hay igualmente una cierta impronta tecnocrática que otorga gran importancia al conocimiento experto a costa de una marginación relativa de las sensibilidades y problemas más inmediatos de los ciudadanos.

Pretender que un nuevo parlamento europeo pueda solventar de un plumazo todos estos problemas introduciendo nuevos aires de transparencia en las líneas de actuación de las instituciones europeas probablemente sea una aspiración muy optimista: pero la experiencia histórica nos indica que los parlamentos han sido siempre los principales instrumentos para impulsar nuevas orientaciones a la actuación de los ejecutivos, intensificando sus mecanismos de control y asegurando una mayor capacidad de adecuación a las necesidades ciudadanas. 


\section{Dimensión economica. ¿Ha pasado el peligro para el euro?}

Pero nuestra percepción de la realidad europea, más que en su dimensión políti$\mathrm{ca}$, parece que ha tenido hasta ahora un sentido predominantemente económico, puesto que al fin y al cabo el mercado común europeo fue el antecedente histórico de la propia Unión.

Sin embargo el considerable avance que en su momento supuso la unión monetaria no fue adecuadamente culminado con unos instrumentos paralelos de política fiscal, permitiendo que no se produjeran desequilibrios ante la posibilidad de choques asimétricos. La existencia de una moneda común junto a unas políticas fiscales diferenciadas constituía un panorama que, como sugirieron en su momento algunas voces, presentaba riesgos de falta de capacidad de respuesta ante coyunturas de crisis que requieren de actuaciones excepcionales que deben proyectarse de forma unitaria. La asunción por parte de Europa de un poder monetario requería seguramente de un paralelo poder fiscal, configurado a partir de una homogeneización de los distintos sistemas estatales, pero implicando una capacidad suficiente para decidir una política común ante situaciones de crisis. Se trata de una serie de desafíos que se han hecho especialmente difíciles tras el intenso proceso de ampliación de la propia Unión.

Recordemos que la crisis financiera y económica internacional generó problemas que no han afectado por igual a todos los países sino especialmente a Grecia, así como Irlanda y Portugal y en menor medida España e Italia. Podríamos afirmar que el propio diseño de la eurozona determinó que los problemas fueran especialmente acusados en estos países; aunque al mismo tiempo los propios problemas internos de estas naciones afectaron al conjunto de la unión monetaria.

En este contexto las decisiones adoptadas a partir del desencadenamiento de la crisis no han conseguido superar la impronta intergubernamental, y con frecuencia las autoridades europeas representadas en el Consejo, más que a los intereses generales de la Unión han parecido mirar a los intereses de sus respectivos estados. Sólo la acuciante gravedad de la crisis financiera ha permitido que la actuación de Mario Draghi al frente del Banco Central haya cosechado un cierto éxito, consiguiendo ahuyentar los fantasmas de una catastrófica suspensión de pagos al reducir las primas de riesgo de la deuda de los estados.

Otras decisiones de mayor calado se refieren a la construcción de la unión bancaria europea, lo que supone un supervisor único así como un fondo común de garantía de depósitos y un mecanismo común para la resolución de las crisis bancarias. 
Pero son mecanismos que se van poniendo en marcha con la tradicional lentitud de la Unión Europea; aunque no cabe duda de que la consecución de la unión bancaria supone un avance no sólo en el proceso de unión monetaria sino también en la consecución de una auténtica unión económica.

Por otra parte resulta discutible que la estrategia restrictiva impuesta por Alemania con el apoyo de algunos estados del norte europeo, constituya la opción más adecuada ante una crisis tan grave, no sólo por los dramáticos efectos sociales que viene generando en los países del sur europeo, sino porque corre el riesgo de convertirse en un obstáculo para la esperada recuperación económica que, aunque a comienzos de 2014 parece estar empezando a notarse, presenta todavía numerosas incertidumbres en los países del sur.

No obstante, la situación actual de la economía irlandesa nos hace pensar que la política de estabilidad quizás haya sido indispensable para corregir sus graves problemas económicos. Pero seguramente la política de austeridad alemana hubiera operado de una forma más adecuada si se hubiera acompañado de otras medidas que estimularan la actividad económica de la zona, lo que hubiera evitado la dramática caída de los ingresos que está reduciendo la capacidad de maniobra de las autoridades estatales ante una crisis cuyas terribles consecuencias sociales en términos de desempleo no podemos olvidar ni un momento.

Pero aún más dramática sería la hipótesis de que, en realidad, el diagnóstico de base desde el cual se han abordado las estrategias de lucha contra la crisis sea en realidad un diagnóstico erróneo y que las propias estrategias de respuesta, en forma de políticas restrictivas de corte neoclásico (inspiradas por el gobierno alemán), acaben produciendo al final efectos contraproducentes: sería como obligar a un enfermo grave a hacer una dieta que lo va a debilitar aún más. Tras este posible error estratégico aparecen finalmente intereses concretos: por ejemplo, las presiones para imponer un rescate a Grecia evitando una suspensión de pagos podrían haber escondido en realidad un intento de los bancos alemanes (principales titulares de la deuda griega) de conseguir la devolución efectiva de sus fondos. Problemas parecidos aparecen en España donde la absorción de la deuda pública ha corrido a cargo de las propias instituciones bancarias, lo que suscita numerosas incertidumbres ante riesgos futuros. Aunque también habría que tener en cuenta opiniones como las del expresidente del BCE Jean Claude Trichet quien considera que el reforzamiento del pacto de estabilidad constituye una condición necesaria de la política económica europea del futuro.

Pero la necesidad de atribuir a la Unión competencias efectivas de política fiscal no se vincula exclusivamente a razones de carácter macroeconómico. Tengamos 
en cuenta que el relativo alejamiento de los ciudadanos europeos respecto de las instituciones de la Unión no se deriva solamente de cuestiones abstractas de pura "pertenencia" sino de aspectos más prosaicos: y es que los ciudadanos europeos no pagamos en rigor de forma directa impuestos europeos. De esta forma el presupuesto europeo se configura como una especie de ajuste de cuentas entre los estados, donde lo que éstos dan a la Unión lo tratan de recuperar "a posteriori" en una especie de juego de suma cero.

Afirmar que la pertenencia ciudadana a una determinada instancia institucional dependa al final del hecho de que tengamos que sacar dinero de nuestra cartera, puede resultar una visión algo dura o escéptica: pero es evidente que, al pagar impuestos, nos hacemos auténticos ciudadanos responsables y nos sentimos al mismo tiempo con capacidad para exigir, controlar e influir sobre las instituciones a las que sufragamos con nuestro dinero.

Las ideas difusamente sugeridas ante los medios de comunicación por José Manuel Durão Barroso acerca de un nuevo tratado capaz de consolidar y reforzar a las instituciones de la Unión, parecen insertarse en una trayectoria de continuidad histórica que en última instancia conduciría hacia un reforzamiento institucional de la Unión.

En resumen, habría que plantearse si ya están resueltos los problemas de la eurozona. $Y$ en este sentido conviene tener presentes varios riesgos que, además de bloquear la solución de dichos problemas, van a determinar el propio futuro de la Unión Europea: las dificultades de la economía griega, que todavía no se han conseguido superar; la lentitud en la adopción de decisiones relacionadas con la unión bancaria; la falta de acuerdo para adoptar decisiones comunes de política fiscal (por ejemplo, en lo relativo a la emisión de eurobonos); así como al ascenso de posturas populistas de cara a las próximas elecciones, que en parte se ven apoyadas por la mejor posición relativa conseguida por la economía del Reino Unido en contraste con el resto de la unión monetaria.

\section{Europa ante el mundo}

Pero en pleno siglo XXI la percepción de Europa no debe reducirse a una pura visión endógena pensada tan sólo para la resolución de nuestros propios problemas "domésticos". Como ha recordado Habermas en su jAy! Europa (2009), el nuevo contexto mundial implica que sin la colaboración internacional ya no puede 
garantizarse la seguridad física en el propio territorio ante los riesgos derivados de la tecnología, el crimen organizado, el nuevo terrorismo, o, en otro orden de cosas, los efectos de las corrientes migratorias. Los propios sistemas jurídicos nacionales y los procedimientos de formación y control de la voluntad democrática se ven interferidos por las disposiciones y regulaciones internacionales limitando el margen de intervención de los propios gobiernos.

Desde esta perspectiva no debemos perder de vista el hecho de que, al menos hasta hace muy poco, Europa era también un modelo de referencia en el escenario mundial, es decir, un espejo en el que trataban de mirarse otros pueblos y otros países. Sin embargo parece cierto que, en una dimensión macroeconómica de proyección mundial, la posición europea ha perdido posiciones en los últimos años: y así se viene afirmando que el eje de gravedad del planeta hace tiempo que se ha alejado de la vieja Europa. El auge de algunas economías emergentes como Brasil o México, la explosión de China o de la India, la capacidad innovadora norteamericana, el consistente apogeo del Pacífico, junto a otros muchos factores, parecen haber dejado a nuestro viejo continente sumergido en un marasmo histórico, sin capacidad de respuestas para hacer frente a la crisis. Desde esta perspectiva parecería como si Europa ya sólo interesara a nivel mundial como un sitio tranquilo y agradable para hacer turismo cultural: un confortable rincón de encuentro de viejas historias, hermosos museos, obras de arte y brillantes monumentos. Y ello a pesar de que, hasta ahora, la Unión Europea sigue constituyendo la experiencia más innovadora de avance en un modelo de construcción política de dimensión supraestatal.

Absorbidos ante la vorágine de la crisis económica y sus dramáticas consecuencias sociales, seguramente nos olvidamos a veces de lo que ha representado Europa ante el mundo, en el sentido de un espacio de convivencia inspirado en valores de solidaridad, libertad y tolerancia. Valores que al fin y al cabo se conectan con la propia tradición cristiana, por más que tal referencia no se introdujera finalmente en el Tratado de Lisboa (en realidad, se trataba de una decisión estratégica probablemente orientada a permitir una eventual entrada de Turquía en la UE). Un marco de convivencia que ha permitido a lo largo del tiempo una adecuada integración entre esferas públicas, económicas y sociales, que se expresa en el ideal histórico del denominado Estado social.

Lo paradóiico es que, a pesar de ser Europa la cuna del Estado social, en la práctica los estados se han venido resistiendo a transferir a la Unión sus ámbitos de poder más relacionados con las políticas de bienestar y asistencia social: seguramente por tratarse de los sectores que generan un mayor grado de legitimidad y también 
de rentabilidad electoral. Es posible que esta brecha sea la que explique algunas de las claves de la gran crisis de la Unión, en la medida en que la defensa del Estado social parece una responsabilidad europea que, sin embargo, no está en las manos de las propias instituciones de la Unión.

Por eso, cuando se habla del desplazamiento del eje de gravedad hacia otras zonas del planeta o hacia el eje del Pacífico, debemos ser capaces de mantener una cierta perspectiva crítica: la afirmación, acaso excesivamente simplificada, de que el proyecto europeo está fracasado, parece implicar que ha debido surgir otro modelo mejor en algún lugar del planeta. Es decir, que el ideal del estado de bienestar ha sido "desbordado" por otros modelos históricamente más innovadores o avanzados, que acaso se focalizan en otros ámbitos como las nuevas tecnologías, en sistemas alternativos de desarrollo económico, o en nuevas formas de dinamismo social o cultural.

Sin embargo, un análisis realista de la situación de estos países emergentes nos permite descubrir que, en rigor, no existe un auténtico modelo alternativo al Estado social. Más bien comprobamos, incluso, que esos países emergentes parecen aspirar a ser reproducciones más eficientes del mismo modelo que surgió en la vieja Europa a partir de mediados del siglo XX. O sea que, de mayores, quieren ser como nosotros.

Reivindicar un modelo de sociedad inspirado en valores sigue constituyendo seguramente un desafío dramáticamente reactualizado para los ciudadanos europeos víctimas de la crisis. Pero debemos conservar el suficiente nivel de conciencia crítica como para entender que se trata al mismo tiempo de una aspiración compartida por todos los hombres de buena voluntad de otras partes del mundo. Más allá de falsas visiones "eurocéntricas" seguramente tenemos el deber de entender que estamos tratando de mantener y de recuperar un modelo de Estado social que sigue constituyendo el principal punto de referencia para la humanidad. Y que como electores en los comicios de mayo de 2014, alguna responsabilidad tendremos todos en ello.

Por eso es tan importante que en las elecciones europeas tratemos de superar visiones limitadas o egoístas, centradas en los problemas internos de cada estado, para tratar de asumir una condición de ciudadanía europea, que al mismo tiempo puede constituir también en cierto modo una especie de ciudadanía mundial desde la cual sea posible avanzar hacia modelos equilibrados de globalización en la escala planetaria. 